\title{
Laser therapy penetration depth: a near-infrared study on a horse tendon model
}

\author{
Monica Monici ${ }^{1}$ \\ Alessio Gnerucci ${ }^{2}$ \\ Tatjana Falconi ${ }^{3}$ \\ Daniele Bani ${ }^{4}$ \\ Francesca Cialdai ${ }^{1}$ \\ Franco Fusi ${ }^{2}$ \\ Giovanni Romano² \\ 1 ASAcampus Joint Laboratory, ASA Res. Div. \& \\ Dept. of Clinical and Experimental Biomedical \\ Sciences "Mario Serio", University of Florence, \\ Florence, Italy \\ 2 Department of Experimental and Clinical Biomedical \\ Sciences "Mario Serio", University of Florence, Italy \\ ${ }^{3}$ MDV, Equine practitioner, S. Felice del Benaco, \\ Brescia, Italy \\ 4 Department of Clinical and Experimental Medicine, \\ University of Florence, Florence, Italy
}

\section{Corresponding author:}

Alessio Gnerucci

Department of Experimental and Clinical Biomedical Sciences "Mario Serio",

University of Florence

Viale G. Pieraccini 6

50139 Florence, Italy

E-mail: alessio.gnerucci@unifi.it

\section{Summary}

Introduction: Laser therapy is widely used in the management of tendon diseases and injuries, being effective in reducing pain and promoting tissue repair. However, laser transmission properties in the different biological tissues are not completely known. Our aims were: 1) define a method to model and measure the penetration depth (PD), a fundamental optical parameter describing laser transmission in tissues; 2) verify whether PD depends on the tendon type.

Methods: Ex-vivo specimens (horse) were collected from the superficial flexor (SDFT) and suspensory tendons (SL). A dual wavelength near-infrared laser source was used. Laser transmission through tendon samples was measured using an optical bench. The PD was estimated by the Lambert-Beer equation modeling.

Results: PD values are $\sim 0.16 \mathrm{~mm}$ for SDFT and $\sim 0.5 \mathrm{~mm}$ for SL, respectively, corresponding to a respective power attenuation factor of $\sim 10^{-3}$ and $\sim 10^{-1} \mathrm{~mm}^{-1}$.
Conclusion: Different tendon types show very different PD values. Knowledge of PD allows to quantitatively estimate the light power arriving at any depth in different tissues and biological structures. This methodology is not limited to the tendon case nor to near-infrared sources, allowing for a better understanding of the physical basis of laser therapy, improvement of treatment protocols and dose definition.

KEY WORDS: near-infrared radiation, light propagation, tendinopathies, Lambert-Beer equation.

\section{Introduction}

Laser therapy, alone or joined with other therapies, is widely used as a valid support in the management of tendon diseases by controlling the inflammation while promoting a better healing. However, literature presents few studies on the propagation of laser radiation into tendons alone, concentrating more on whole anatomical structures where tendons are present ${ }^{1-3}$. Despite common features of dense regular connective tissue structures, tendons show remarkable differences in their morphological, molecular and mechanical properties which relate to their specialized function and to the magnitude of strain that the tendon normally experiences ${ }^{4}$. The structural differences, mostly arising from changes in matrix composition and organization, result in different optical properties ${ }^{5,6}$.

Tendon injury is also a much important topic in veterinary medicine, being one of the most common causes of wastage in performance horses. The most frequently observed injury is the intrinsic or strain injury which predominantly affects the palmar soft tissue structures supporting the metacarpophalangeal joint, in particular the superficial digital flexor tendon (SDFT), but also the suspensory ligament (SL) and accessory ligament of the deep digital flexor tendon (ALDDFT). These structures are classified as weight bearing tendons, such as the Achilles tendon in humans, whose model is often represented by SDFT. To this regard, the horse represents an attractive large animal model not only for equine related studies but for research on human tendon injury, due to the shared characteristics of aging phenotypes ${ }^{7}$, elastic energy storing function common to the weight-bearing tendons of both species ${ }^{8,9}$ and injury induced by natural athletic activity. Horse SDFT, SL and ALDDFT are particularly prone to injury: most of ten- 
don injuries (97-99\%) occur to the forelimb tendons. SDFT injuries are common in all disciplines of competing horses. The severity of damage goes from fibrillar slippage through individual fibre rupture to fascicule rupture and, finally, complete rupture. Injured ligaments heal by the normal reparative processes including inflammation with removal of injured tissue, proliferation and migration of fibroblasts, which produce collagenous tissue and ligament remodelling ${ }^{10}$. Tendons can be damaged by intrinsic strain and, more rarely, by extrinsic damage such as penetration. The usual tendinopathies in the SDFT arise by overstrain from sudden over-extension of the metacarpophalangeal joint, but most commonly from a preexisting degeneration consequent to the chronic damage due to loading closed to its structural limit.

Tendinopathies still represent a great challenge in both human and veterinary medicine and many treatments have been proposed over the last decades with poor prognosis for returning to previous athletic activity for a long time without re-injury. Treatment of tendinopathy needs prolonged time out of training and controlled exercise program associated with ultrasound monitoring to assess the healing process and, consequently, increase exercises. The controlled exercise can or can't be associated with traditional treatments such as blistering (in case of horses), local or systemic injection (Hyaluronan, Polysulfated Glycosaminoglycan), physical therapies (laser radiation, therapeutic ultrasound, extracorporeal shock wave) and, more recently, regenerative treatments such as Platelet Rich Plasma (PRP) and Mesenchimal Stem Cells (MSC), prevalently derived from bone marrow or fat and injected into the lesion under ultrasonographic guide ${ }^{11,12}$.

Surgical treatments are also applied. The choice of the surgical procedure is related to the type and location of the lesion ${ }^{13}$. A persistent inflammatory stimulus, with sustained production of growth factors, proteolytic enzymes and cytokines, is a common feature of fibrotic diseases, stimulating the deposition of connective tissue elements that are detrimental to normal tissue architecture. Fibrosis is the final common pathogenic process for many forms of chronic inflammatory diseases. Although the deposition of scar tissue is an integral component of the healing response, the altered composition of the tendon ECM compromises the structural and functional properties of tendons and potentially contributes towards the high risk of reinjury ${ }^{14}$.

Physical therapies are often used as part of the therapeutic strategy for tendinopathies ${ }^{15}$. Among them, the most extensively studied for its effectiveness in tendinopathies is laser therapy, often applying low power lasers but, more recently, also using high power devices. Laser therapy, alone or joined with other therapies, was proposed as a valid support in the management of tendon lesions by controlling the inflammation while promoting a better healing.

Although the effectiveness of laser therapy in reducing inflammation ${ }^{16-18}$, favouring healing and prevent- ing fibrotic scar formation ${ }^{19}$ has been demonstrated in a number of applications, very few studies concerning the application to horse tendonithis have been published and clinical studies on tendonithis in humans present controversial results 20,21 . It is known that studies on laser therapy are difficult to compare because different sources, wavelengths, treatment parameters and modalities are used. Moreover, also anatomical sites and characteristics of the treated tendons differ (shoulder, elbow, leg, etc.). Therefore, it is not surprising that inconsistent results are reported.

When a laser beam impinges on a biological structure, the propagation of radiation into the tissues and the resulting effects depend on both the tissue properties and irradiation parameters, such as wavelength, power and emission modality (i.e. whether continuous wave or pulsed, and, in the latter case, pulse characteristics), exposure time, etc. ${ }^{22-24}$.Therefore, the information on laser source and tissue characteristics, that is optical, thermal and mechanical properties, enable to model and predict laser-tissue interaction phenomena and ultimately to select specific applications and improve the effectiveness of the therapy. In literature, some studies on the characterization of the optical properties of animal tendons may be found 25,26 . Due to the presence of collagen, the tendon tissue may be imaged and studied also by refined but complex and expensive techniques, such as second harmonic generation ${ }^{25}$ or elastic scattering spectroscopy 26 .

In this context, the aim of the present study was to define a simple method for the modelling and measurement of near infrared (NIR) laser light penetration into tendon structures involved in the most frequent tendon lesions in the horse. The laser source was a commercial NIR system $\left(\mathrm{MLS}^{\circledR}\right)$, widely used in both human and veterinary medicine. The outcomes of this study can help to better understand the physical basis of laser therapy and improve treatment protocols for application to the management of tendon injuries both in humans and animals, notably horses.

\section{Materials and methods}

\section{Tendon specimens}

The forelimbs of an adult non-thoroughbred horse of around $500 \mathrm{~kg}$ weight, without tendon injuries, were obtained from the slaughterhouse. Individual tendon specimens were obtained from the horse metacarpal region.

With the use of a scalpel, the skin was removed from the carpus to the foot, then the SDFT and SL were isolated. They were raised from the surrounding tissues. The portions from the carpus to the fetlock of the tendons and from the proximal insertion of the SL to the distal insertion of the two branches on the sesamoid bones were taken and frozen $\left(-80^{\circ} \mathrm{C}\right)$. Frozen specimens from the horse tendons $\left(\sim 0.5 \mathrm{~cm}^{3}\right)$ previously cut by a scalpel, were mounted in cryostat 
embedding medium (Killik, Bio-Optica, Milan, Italy) onto metal support plates, snap-frozen and cut into sections of different thickness with a cryostat (CM 1950 , Leica Microsystems, Milan, Italy) at $-20^{\circ} \mathrm{C}$. The sections were then collected on glass slides, air-dried and kept at $-20^{\circ} \mathrm{C}$ until use. In tendons, the collagen fibers are almost oriented in one direction (i.e. the longitudinal one) and the tissue shows a very high mechanical strength in that direction. In other words, the orientation of the fibers is responsible for tendon anisotropy, both from the morphological and mechanical point of view. To consider the effect of tendon anisotropy on the optical properties, all samples were sectioned both transversally and longitudinally respect to the main fiber direction. For each sample and orientation, slices of 10,20 and $50 \mu \mathrm{m}$ thickness were obtained to perform the optical measurements. Due to their small volume, frozen sections rapidly arrived at room temperature $\left(\sim 25^{\circ} \mathrm{C}\right)$ before the beginning of the measurement. Possible sample drying (easily recognized by a change in the slice transparency) was avoided by rehydration with physiological solution, as the water content in the sample may influence its optical properties.

\section{Laser source}

The laser source was a Multiwave Locked System laser (MLS ${ }^{\circledR}$, ASA Srl, Vicenza, Italy). It is a commercially available laser source built in compliance with EC/EU rules, which received FDA approval and is widely used in clinics. MLS laser is a class IV NIR laser with two synchronized sources (laser diodes). These emit at different wavelengths, peak power and emission mode. The first one is a pulsed $905 \mathrm{~nm}$ laser diode with, each pulse (25 W peak optical power) being composed of a pulse train (100 ns single pulse width, $90 \mathrm{kHz}$ frequency). The frequency of the pulse trains may be varied in the range $1-2000 \mathrm{~Hz}$, thus varying the average power delivered to the tissue. The second laser diode $(808 \mathrm{~nm})$ may operate in continuous (maximum power $1.1 \mathrm{~W}$ ) or pulsed mode (repetition rate $1-2000 \mathrm{~Hz}, 550 \mathrm{~mW}$ mean optical power), with a $50 \%$ duty ratio independently of the repetition rate. The two laser beams are emitted synchronously and the propagation axes are coincident.

\section{Optical measurements}

The parameter of interest of our measurements was the laser penetration depth $(p)$. Briefly, it can be defined as the distance into the sample after which the laser power decreases of a factor $e$, in the hypothesis of an exponentially decreasing power (see eq. 1). From a practical point of view, this means that the laser power at a depth $=p, 2 p$ or $3 p$ is reduced to about $37 \%, 13.5 \%$ and $5 \%$ of its value at the tissue surface, respectively, independently on the impinging laser power. To obtain the value of $p$, the following measurements were performed: (i): the laser power transmitted by a given tendon section (I); (ii): the laser power measured in absence of the sample, representing the laser power impinging on the tendon section $\left(I_{0}\right)$; (iii) the power measured by the sensor with the laser off, representing the spurious ambient light to be subtracted from each of the previous measurements $\left(I_{a}\right)$; (iv): the laser power transmitted by the glass slide only $\left(I_{g}\right)$. This quantity resulted negligible respect to the previously defined ones (data not shown), so that it was neglected in further calculations. As a final step $I, I_{0}$ and $I_{a}$ were combined to obtain $p$ as described in the Mathematical model section.

Optical measurements were performed by exposure of each tendon sample to the MLS ${ }^{\circledR}$ source, contemporarily measuring the optical power transmitted by the sample. For this purpose, a specific setup was assembled (Fig. 1).

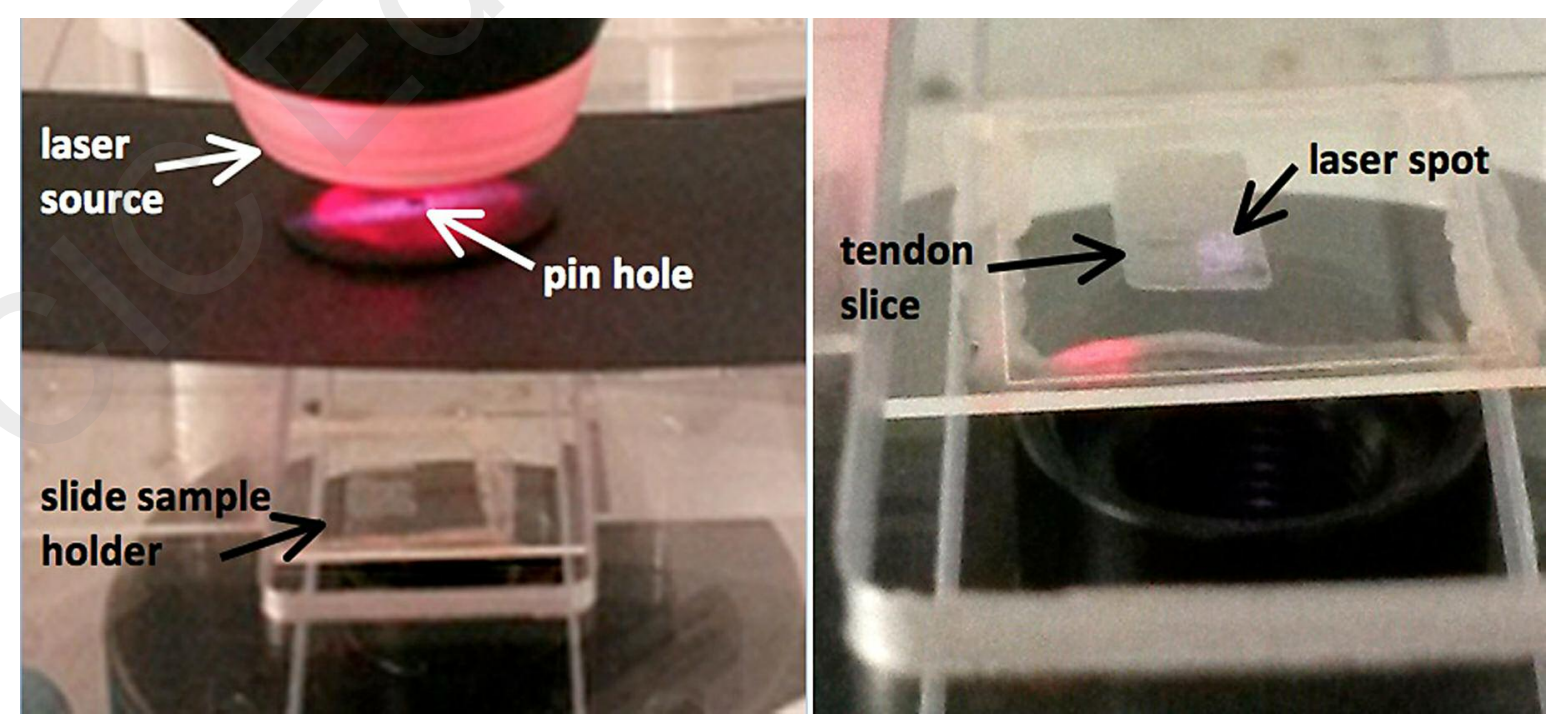

Figure 1. The optical setup realized for the light transmission measurements. Left panel. Detail of the laser source, the pin hole and the sample holder. Right panel. Detail of the laser spot on the tendon slice sample. 
The laser source was positioned above the sample at $\sim 5 \mathrm{~cm}$, impinging on it vertically. To obtain a small and reproducible illumination area ( $2 \mathrm{~cm}$ diameter) at the sample level, a $2 \mathrm{~mm}$ diameter pin hole was inserted just above the sample $(\sim 1 \mathrm{~cm}$ distance). To measure the optical power, a pyroelectric detector (PC Link, laserpoint, Italy) was positioned immediately below the sample (see ${ }^{1}$ ). By means of a smartphone camera (CMOS sensor, Samsung) sensitive to NIR radiation, the laser spot on the sample surface was imaged for each slice, to verify that no portion of the beam was out of the sample and the laser spot impinging on the sample was centered on the pyroelectric sensor surface.

The laser was set up with the following parameters: continuous emission mode; power percentage $=50 \%$ (relative to the maximum output power), pulse train repetition frequencies: $10 \mathrm{~Hz}$. For each tendon section, 5 measurements were collected by moving the sample respect to the laser beam, to illuminate different regions of the same section. By calculating the average and standard deviation of these measurements, variations of the tissue optical and morphological properties along the section surface were considered.

\section{Mathematical model}

Light-tissue interaction was modeled by the LambertBeer (LB) law, that adopts a plane wave geometry for the incident light beam propagating along the vertical $z$ axis and encountering the sample at $z=0$ (Fig. 2).

The LB equation assumes that the energy loss (dl) due to the passage through the sample thickness $d z$ is proportional to $d z$ and to the intensity at the $z$ level, namely: $d l \propto l(z) d z$, being $l(z)$ the intensity at $z$, so that:

$$
I(z)=I(0) e^{-z / p}
$$

where $p$ is the laser penetration depth that takes into

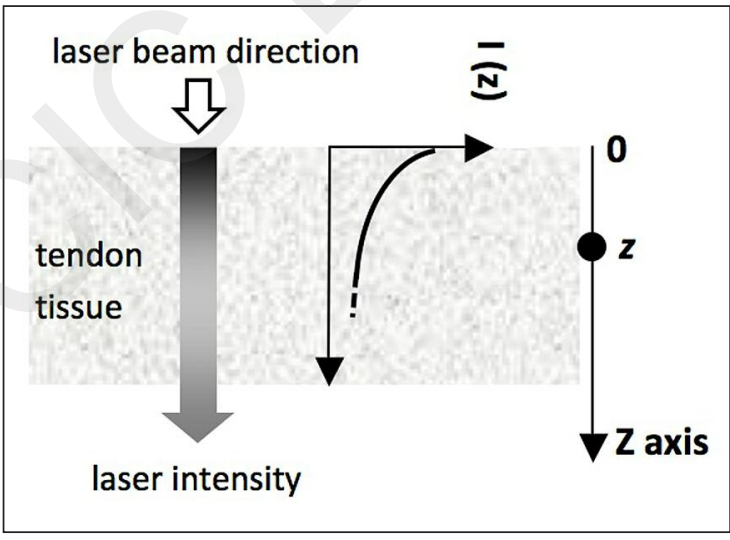

Figure 2. Schematic view of the laser-tissue interaction geometry. The exponential decrease of light intensity I(z) vs z (vertical coordinate) is also shown. account the contribution of both absorption and scattering defining the so called laser beam extinction. By adopting the LB model it is possible to measure $p$ starting from the measurements described in the previous section:

$$
p=\frac{z}{\ln \left(\frac{I_{0}-I_{a}}{I-I_{a}}\right)}
$$

where $\left(I-I_{a}\right)$ and $\left(I_{0}-I_{a}\right)$ represent $I(z)$ and $I(0)$ respectively and $z$ the sample (i.e. the tendon section) thickness. This approach allows to measure the $p$ value with a single tendon section measurement. In this work, it was preferred to adopt a more robust approach that consisted in obtaining the curve $\left(I-I_{a}\right)$ as a function of the sample thickness $z$ (see next section, Figs. 3, 4) thus using all the measurements for the three chosen sections $(10,20,50 \mu \mathrm{m})$. This curve was then fitted with the exponential LB equation (1) thus recovering the best fit parameter $p$. Once $p$ is known, another parameter can be calculated, namely the "laser attenuation factor" (a). This corresponds to the factor of attenuation for the laser power arriving at $1 \mathrm{~mm}$ depth (respect to the impinging laser power), and in general to the attenuation that the laser power experiences for every $\mathrm{mm}$ of penetration into the tissue. For example, if $\propto=10^{-1}$ it means that, for every $\mathrm{mm}$ into the tissue, the laser power is reduced of $90 \%$ (corresponding to a $10 \%$ power arriving at $1 \mathrm{~mm}$ depth) so that after e.g. $2 \mathrm{~mm}$ the power is reduced to $1 \%$ of its initial value.

\section{Results}

The measurements for the SDFT and SL are presented in Figures 3 and 4, respectively. The best fit estimates for the penetration depth are summarized in Table I.

\section{Discussion}

The study of light-tissue interaction is of paramount importance to better understand the physical principles at the basis of the therapeutic action of laser radiation. The increase of knowledge about light propagation into different tissues can help to prepare guidelines for laser application in human and veterinary medicine, to improve treatment protocols through the choice of suitable laser parameters and thus, ultimately, to increase laser therapy effectiveness.

When studying the application of laser therapy in human and veterinary medicine, it should be considered that: i) different tissues have different optical properties; ii) the properties of a given tissue type, e.g. skin, can change depending on the animal species and skin phototype; iii) the properties of a tissue or anatomical structure can change even within the same organism, depending on the function and body district considered, as confirmed in this study. 

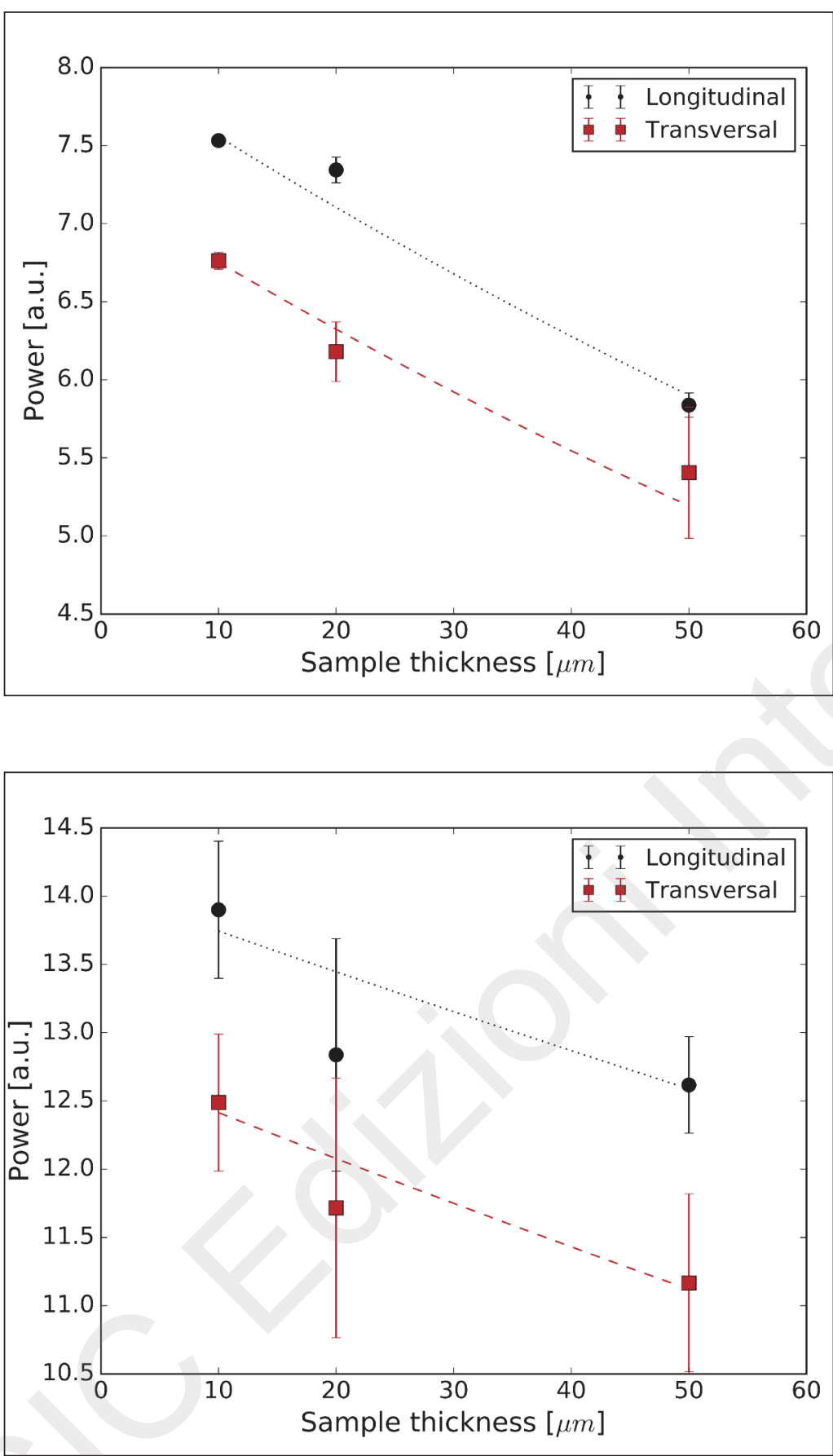

Figure 3. SDFT specimen results. Transmitted laser power vs sample thickness. Circles: Iongitudinal sections. Squares: transversal sections. Dotted and dashed lines represent Lambert-Beer best fit model to longitudinal and transversal section case, respectively.

Figure 4. SL specimen results. Transmitted laser power vs sample thickness. Circles: longitudinal sections. Squares: transversal sections. Dotted and dashed lines represent LambertBeer best fit model to longitudinal and transversal section case, respectively.
In the case of tendons, literature reports result on the penetration of visible / near-infrared light in the rat model ${ }^{1,27}, 780 \mathrm{~nm}$ radiation in the pig model ${ }^{25}$ and very few in human tendons with refined but complex techniques, such as optical coherence tomography ${ }^{28}$. Studies on visible light penetration in the horse tendon ${ }^{2,3}$ report measurements performed directly on the whole leg. In such an experimental setting, the obtained results are strictly linked to the specific experimental conditions (not only laser parameters but also animal size, thickness of the various tissues penetrat- ed by the laser, environmental light conditions, etc.), therefore they cannot be generalized to characterize the physical parameters describing light penetration into tendons or different types of biological tissues. Moreover, when the light absorption is very high (close to 100\%), as in the case described in Ryan et al. ${ }^{3}$, the measured intensity of the exiting light can be comparable to the measurement error.

Rather than simply measuring the light transmitted by a whole anatomical structure (e.g. considering ex vivo or in vivo measurements on the whole horse leg or 
Table I. Penetration depth $(p)$ and attenuation factor $(a)$ for the various tendon samples. Error was estimated as standard deviation of the best fit parameters (Lambert-Beer model).

Superficial flexor

Suspensory ligament

\begin{tabular}{llllc}
\hline & longitudinal & transversal & longitudinal & transversal \\
\hline $\boldsymbol{p}(\mathbf{m m})$ & $0.16 \pm 0.03$ & $0.15 \pm 0.04$ & $0.5 \pm 0.2$ & $0.4 \pm 0.1$ \\
$\boldsymbol{a}\left(\mathbf{m m}^{-1}\right)$ & $2.010^{-3}$ & $1.310^{-3}$ & $1.010^{-1}$ & $6.310^{-2}$ \\
\hline
\end{tabular}

portions of it), we focused on the measurement of the fundamental optical parameters defining the light transmission properties in tissues, in particular the penetration depth $p$. In this respect, we propose an approach based on the use of the penetration depth instead of the depth of penetration, the last being defined as the total length traveled by the laser light through various tissues in the specific case considered (that is the specimen thickness). By measuring the value of $p$ and/or $\alpha$ in the tendon, for a laser of a given wavelength, we can evaluate the laser attenuation due to its passage through a given tendon (in vivo or ex vivo) or also estimate the portion of light power arriving at any given depth in the tendon. Out of a laser attenuation measurement, we can also estimate the laser energy "released" in the tendon structure, which may be related to the therapeutic outcome. This approach offers a general methodology whose applications are much broader and advantageous than many empirical studies found in literature, limited to the mere measurement of the light power emerging from a treated area. In addition, our approach can be considered while comparing e.g. normal and pathologic tissue, where the measurement of fundamental parameters like $p$ or $\alpha$ can be more fruitfully connected to intrinsic changes in the specific tissue structure, rather than a comparison between the percentage of transmitted power by the specific specimen. This, in fact, can be affected by many factors which may be difficult to acknowledge and quantify. In general, the obtained values for $p$ are in line with the findings of Hall et al. ${ }^{27}$, where the value of $\mu=1 / p$ are reported for the mouse tendon tissue. First, we can observe that $p$ values for the longitudinal sections are higher than the transversal ones, which may be due to the different arrangement of the collagen fibers. This is especially interesting if we consider that the longitudinal cut better represents the in vivo treatment conditions. It is worth noting that most part of the error is due to tissue inhomogeneity rather than to instrumental reasons. In fact, it is difficult for the transversal sections to obtain slices with very smooth surfaces and perfectly adherent to the glass slide, that is the optimal condition for optical measurements. Interestingly, the light penetration depth of the two tendons resulted very different, reflecting different structural properties: compared to the SDFT, the SL has lower elastic modulus and mass average fibril diameter, but higher levels of sulphated glycosaminoglycans (GAG), water and cell content. All these structural characteristics affect the optical properties of the tissue.

Finally, the relatively high laser attenuation by the tendon tissue (Tab. I) is not necessarily a "problem" from a therapeutic point of view, as this is associated with a "high" energy release inside a portion of the treated area which, in turn, is correlated with the therapeutic effect. In addition, it should be considered that the energy release induces a biological response, that affects also the surrounding tissues, due to the fact that laser treatment can modulate the release of soluble biochemical factors.

In summary, the penetration depth depends on the tissue structure (once the wavelength is fixed) and the knowledge of this optical property allows for the choice of optimal treatment parameters according to the type and depth of the injury and the anatomical site in which it is located.

In other words, if we know the penetration depth of a specific radiation into a given tendon, or any other anatomical structure, the treatment can be adapted to the type and depth of injury. When in the superficial part of the tendon (or any other anatomical structure) the attenuation, and consequently the energy release, are high, a suitable setting of the treatment parameters (fluence, exposure time, pulse duration and frequency, etc.) is required to increase the energy release in the inner part of the organ.

We propose a methodology to be applied to the study of laser light interaction (regardless of the source type) not only with the tendon tissue, but more generally to any other tissue type, provided the proper theoretical context (LB law) and setup geometry are considered. Once the value of the penetration depth $p$ is known for a specific tissue type, the choice of the irradiation parameters (notably the laser power or power percentage) may be driven accordingly, to deliver the correct amount of light to the tissue(s) to be treated. In fact, it should be considered that a higher $p$ value is associated with a higher power reaching a same depth in the tissue and vice versa, once the emitted laser power is fixed. Therefore, the operator may have interest in raising or lowering the laser power according to the $p$ value measured for the specific tissue being irradiated. From a clinical perspective, this work confirms the importance to know the tissue optical parameters, paramount to define the best treatment conditions and consequently to optimize the therapeutical efficacy of any laser treatment. 


\section{Conflict of interest}

There are no other relationships/conditions/circumstances that present potential conflict of interest.

\section{Ethics}

The Authors declare that this research was conducted following basic ethical aspects and international standards as required by the Journal and recently update $\mathrm{in}^{29}$.

\section{References}

1. Joensen J, Gjerdet NR, Hummelsund S, Iversen V, LopesMartins RAB, Bjordal M. An experimental study of low-level laser therapy in rat Achilles tendon injury. Lasers Med Sci. 2012;27:103-111.

2. Hutchison AM, Beard DJ, Bishop J, Pallister I, Davies W. An Investigation of the Transmission and Attenuation of Intense Pulsed Light on Samples of Human Achilles Tendon and Surrounding Tissue. Lasers in Surgery and Medicine. 2012;44: 397-405.

3. Ryan T, Smith RKW. An investigation into the depth of penetration of low level laser therapy through the equine tendon in vivo. Irish Veterinary Journal. 2007;60(5):295-299.

4. Birch $\mathrm{HL}$. Tendon matrix composition and turnover in relation to functional requirements. Int. J. Exp. Path. 2007;88:241-248.

5. Weinreb JH, Sheth C, Apostolakos J, McCarthy MB, Barden B, Cote MP, Mazzocca AD. Tendon structure, disease, and imaging. Muscles Ligaments Tendons J. 2014 May 8;4(1):6673. eCollection 2014 Jan.

6. Tresoldi I, Oliva F, Benvenuto M, Fantini M, Masuelli L, Bei R, Modesti A. Tendon's ultrastructure. Muscles Ligaments Tendons J. 2013 May 21;3(1):2-6.Doi: 10.11138/mltt/2013.3.1. 002. Print 2013 Jan.

7. Dudhia J, Scott CM, Draper ER, Heinegard D, Pitsillides AA, Smith RK. Aging enhances a mechanically-induced reduction in tendon strength by an active process involving matrix metalloproteinase activity. Aging Cell. 2007;6:547-556.

8. Ker RF, Wang XT, Pike AV. Fatigue quality of mammalian tendons. J. Exp. Biol. 2000;203:1317-1327.

9. Wilson AM, McGuigan MP, Su A, van Den Bogert AJ. Horses damp the spring in their step. Nature. 2001 Dec 20-27;414 (6866):895-899.

10. Dakin SG, Dudhia J, Smith RKW. Resolving an inflammatory concept: The importance of inflammation and resolution in tendinopathy. Vet Immunol Immunopathol. 2014;158(34):121-127.

11. Lui PP, Wong OT. Tendon stem cells: experimental and clinical perspectives in tendon and tendon-bone junction repair. Muscles Ligaments Tendons J. 2012 Oct 16;2(3):163-168. Print 2012 Jul.

12. Muttini A, Russo V, Rossi E, Mattioli M, Barboni B, Tosi U, Maffulli N, Valbonetti L, Abate M. Pilot experimental study on amniotic epithelial mesenchymal cell transplantation in natural occurring tendinopathy in horses. Ultrasonographic and histological comparison. Muscles Ligaments Tendons J. 2015 Mar 27;5(1):5-11. eCollection 2015 Jan-Mar.

13. Wu Y, Lin L, Li H, Zhao Y, Liu L, Jia Z, Wang D, He Q, Ruan D. Is surgical intervention more effective than non-surgical treatment for acute Achilles tendon rupture? A systematic review of overlapping meta-analyses.Int J Surg. 2016 Dec;36(Pt A):305311.Doi: 10.1016/j.jisu.2016.11.014. Epub 2016 Nov 10.
14. Crevier-Denoix N, Collobert C, Pourcelot $P$, Denoix JM, Sanaa M, Geiger D, Bernard N, Ribot X, Bortolussi C, Bousseau B. Mechanical properties of pathological equine superficial digital flexor tendons. Equine Vet J Suppl. 1997:23-26.

15. Buchner HHF, Schildboeck U. Physiotherapy applied to the horse: a review. Equine Veterinary Journal. 2006;38(6):574580.Doi: 10.2746/042516406X153247

16. Albertini R, Aimbire F, Villaverde AB, Silva JA, Costa MS. COX-2 mRNA expression decreases in the subplantar muscle of rat paw subjected to carrageenan-induced inflammation after low level laser therapy. Inflamm Res. 2007;56:228-229. Doi:10.1007/s00011-007-6211-6 (a)

17. Rizzi CF, Mauriz JL, Freitas Correa DS, Moreira AJ, Zettler CG, Filippin LI, Marroni NP, Gonzalez-Gallego J. Effects of Lo w-Level Laser Therapy (LLLT) on the Nuclear Factor (NF)-kB Signaling Pathway in Traumatized Muscle. Lasers in Surgery and Medicine. 2006;38:704-713.

18. Monici M, Cialdai F, Ranaldi F, Paoli P, Boscaro F, Moneti G, Caselli A. Effect of IR laser on myoblasts: a proteomic study. Molecular Biosystems. 2013;9:1147-1161. ISSN: 1742-2051. Doi:10.1039/ C2MB25398D

19. Fillipin LI, Mauriz JL, Vedovelli K, Moreira AJ, Zettler CG, Lech O, Marroni NP, Gonzalez-Gallego J. Low-Level Laser Therapy (LLLT) Prevents Oxidative Stress and Reduces Fibrosis in Rat Traumatized Achilles Tendon. Lasers in Surgery and Medicine. 2005;37:293-300.

20. Andres BM and Murrell GAC. Treatment of Tendinopathy. What Works, What Does Not, and What is on the Horizon, Clin Orthop Relat Res. 2008;466:1539-1554. Doi:10.1007/s11999008-0260-1

21. Rees JD, Wilson AM and Wolman RL. Current concepts in the management of tendon disorders. Rheumatology. 2006;45: 508-521. Doi:10.1093/rheumatology/kel046

22. Thomsen S. Pathologic analysis of photothermal and photomechanical effects of laser-tissue interactions. Photochem Photobiol. 1991;Jun;53(6):825-835.

23. Niemz MH. Laser - Tissue Interactions, Berlin, DE: SpringerVerlag. 1996.

24. Rossi F, Pini R, Monici M. Direct and indirect photomechanical effects in cells and tissues. Perspectives of application in biotechnology and medicine. In: Monici M and van Loon J eds. Cell mechanochemistry. biological systems and factors inducing mechanical stress; such as light, pressure and gravity. Trivandrum: Research Signpost/Transword Research Network; ISBN: 9788178954585. 2010:285-301.

25. Lau TY, Ambekar R, Toussaint KC. Quantification of collagen fiber organization using three-dimensional Fourier transformsecond-harmonic generation imaging. OSA-OPTICS EXPRESS. 2012;20:19:21821-21832. Doi:10.1364/OE.20. 021821.

26. Kostyuk O, Birch HL, Mudera V and Brown RA. Structural changes in loaded equine tendons can be monitored by a novel spectroscopic technique. J Physiol. 2004;554;3:791-801.

27. Hall G, Jacques SL, Eliceiri KW, Campagnola PJ. Goniometric measurements of thick tissue using Monte Carlo simulations to obtain the single scattering anisotropy coefficient. Biomed Opt Express. 2012;3(11):2707-2719.

28. Bagnaninchi PO, Churmakov D, Bonesi M, Yang Y, Phelan C, Maffulli N, Meglinski I, El Haj A. Optical properties of human tendons characterized by PSOCT and their relation to tendinopathy: A clinical study. Proc. SPIE 6854, Optical Interaction with Tissue and Cells XIX, 68541C (February 12, 2008). Doi:10.1117/12.763156

29. Padulo J, Oliva F, Frizziero A, Maffulli N. Muscles, Ligaments and Tendons Journal - Basic principles and recommendations in clinical and field science research: 2016 update. MLTJ. 2016;6(1):1-5. 